Journal of Applied AnALysis

Vol. 11, No. 1 (2005), pp. 81-94

\title{
EXISTENCE FOR SOME QUASILINEAR ELLIPTIC SYSTEMS WITH CRITICAL GROWTH NONLINEARITY AND $L^{1}$ DATA
}

\author{
N. ALAA, F. MAACH and I. MOUNIR \\ Received July 18, 2001 and, in revised form, December 10, 2003
}

\begin{abstract}
We prove the existence of weak solutions for some quasilinear elliptic reaction-diffusion systems with Dirichlet boundary conditions and satisfying to the two main properties: the positivity of the solutions and the balance law. The nonlinearity we consider here has critical growth with respect to the gradient and the data are in $L^{1}$.
\end{abstract}

\section{Introduction}

The aim of this work is to study existence of weak solutions for the following quasilinear elliptic system:

$$
\begin{cases}-\Delta u=-f(x, u, v, \nabla u, \nabla v)+F(x) & \text { in } \Omega \\ -\Delta v=f(x, u, v, \nabla u, \nabla v)+G(x) & \text { in } \Omega \\ u=v=0 & \text { on } \partial \Omega\end{cases}
$$

2000 Mathematics Subject Classification. 35J20, 35J25, 35J65, 45H15.

Key words and phrases. Elliptic, quasilinear, quadratic, weak solutions, $L^{1}$ data.

This work was supported by the "Action Intégrée MA/02/33" program, between the University Cadi Ayyad FST Gueliz of Marrakech-Morocco and the ENS Cachan-Bretagne of Rennes-France.

ISSN 1425-6908（C) Heldermann Verlag. 
where $\Omega$ is an open bounded set of $\mathbb{R}^{N}, N \geq 1$, with smooth boundary $\partial \Omega$. $f: \Omega \times \mathbb{R}^{2} \times \mathbb{R}^{2 N} \rightarrow[0,+\infty)$ is a nonlinear function which has critical growth with respect to the gradient. $F, G: \Omega \rightarrow[0,+\infty)$ are non-negative integrable functions.

We are interested in the case where the data are nonregular and the growth of the nonlinear term $f$ with respect to the gradient is quadratic. To help understand the situation, let us mention some previous works concerning systems of the form

$$
\begin{cases}-\Delta u=f(x, u, v, \nabla u, \nabla v)+F(x) & \text { in } \Omega \\ -\Delta v=g(x, u, v, \nabla u, \nabla v)+G(x) & \text { in } \Omega \\ u=v=0 & \text { on } \partial \Omega .\end{cases}
$$

In general such systems have been studied under the so-called "triangular structure", namely

$$
\left\{\begin{array}{l}
f(x, u, v, p, q)+g(x, u, v, p, q) \leq L_{1}(u+v+1), \\
f(x, u, v, p, q) \leq L_{2}(u+v+1), \\
\text { for all }(u, v, p, q) \in \mathbb{R} \times \mathbb{R} \times \mathbb{R}^{2} \times \mathbb{R}^{2} \text { and a.e. } x \in \Omega, L_{1}, L_{2} \geq 0 .
\end{array}\right.
$$

With the boundary conditions and the first inequality in the triangular structure (which is called balance law or mass control), one can derive an $L^{1}$ estimate on the components of the solution. But in general this is not sufficient to prove existence and then additional hypotheses are required. We refer the reader to [11], [12], [16] and the references there in for the semilinear case ( $f$ and $g$ dot not depend on the gradient). In the quasilinear case ( $f$ and $g$ depend on the gradient), the existence has been obtained in [16] when $g=-f$, the data are not regular and the dependence of the nonlinear term with respect to the gradient is subquadratic. This result has been generalized later in [3]. The authors considered the case where $f$ and $g$ satisfy the triangular structure, the data are not regular, and the growth with respect to the gradient is quadratic for the first component.

It is worth to recall here that the parabolic version of these systems has been extensively studied, see [13], [17], [21] et al for the semilinear case and [8], [2] for the quasilinear case.

Let us also point out that the triangular structure played an important role in the study of such systems. It may indeed happen, as it is proved in [18] and [19], that the solutions blow up in finite time if this condition is not fulfilled.

This could justify the conditions on the nonlinear terms we are considering here. Moreover the structure of system (1) leads us to introducing the 
function $w$ solution of the linear problem

$$
\begin{cases}-\Delta w=F+G & \text { in } \Omega, \\ w=0 & \text { on } \partial \Omega .\end{cases}
$$

Then solving problem (1) is equivalent to solve the equation

$$
\begin{cases}-\Delta u+f(x, u, w-u, \nabla u, \nabla w-\nabla u)=F & \text { in } \Omega, \\ u=0 & \text { on } \partial \Omega\end{cases}
$$

and set $v:=w-u$.

Let us now make some precise statements on a model problem like

$$
\begin{cases}u, v \in W_{0}^{1, p}(\Omega) & \\ -\Delta u=-u|\nabla v|^{p}+F(x) & \text { in } \Omega \\ -\Delta v=u|\nabla v|^{p}+G(x) & \text { in } \Omega,\end{cases}
$$

where $|\cdot|$ denotes the $\mathbb{R}^{N}$-euclidian norm and $p \geq 1$.

If we apply the same transformation to this problem, we obtain the equation

$$
\left\{\begin{array}{l}
u \in W_{0}^{1, p}(\Omega) \\
-\Delta u+u|\nabla w-\nabla u|^{p}=F \quad \text { in } \Omega .
\end{array}\right.
$$

If $F$ and $G$ are regular $\left(F, G \in W^{1, \infty}(\Omega)\right)$, the method of sub- and supersolution can be used to prove existence in (4). For instance $u_{1}=0$ is a subsolution and $u_{2}=w$ is a supersolution, then (5) has a solution $u \in W_{0}^{1, \infty}(\Omega) \cap W^{2, p}(\Omega) \forall p<\infty$, see Lions [14].

If $F, G \in H^{-1}(\Omega)$ and $p \leq 2$ then $|\nabla w|^{p} \in L^{1}(\Omega)$. Many authors dealt with this problem and showed that (5) has a solution $u \in H_{0}^{1}(\Omega)$ see [6], [7] and the references there in, see also [4] for the case of data measure.

For our purpose, we are particularly interested in situations where $F$ and $G$ are not regular $\left(F, G \in L^{1}(\Omega)\right)$ and $p \geq 1$. In this case $|\nabla w|^{p}$ do not belong necessarily to $L^{1}(\Omega)$. To overcome this difficulty, we will see how we will proceed to adapt the techniques used in [7] to the resolution of our problem.

We have organized the paper as follows. In Section 2 we give the precise setting of the problem and state the main result. In Section 3 we present an approximate problem and we give suitable estimates to prove that (3) has a solution in the case where the growth of $f$ with respect to the gradient is quadratic. 


\section{Assumptions and statement of main results}

Let $f: \Omega \times \mathbb{R}^{2} \times \mathbb{R}^{2 N} \rightarrow[0,+\infty)$ be a caratheodory function (that is $f$ is measurable with respect to $x \in \Omega$, and $f$ is continuous with respect to $u, v, p, q$ in $\mathbb{R}^{2} \times \mathbb{R}^{2 N}$ ) which satisfies the following assumptions:

$$
\begin{aligned}
& f(x, u, v, p, q) \geq 0 \text { a.e. } x \in \Omega \text { and for all } u, v \geq 0, p, q \in \mathbb{R}^{N} \\
& |f(x, u, v, p, q)| \leq C(|u|+|v|)\left[|p|^{2}+|q|^{2}+K(x)\right]
\end{aligned}
$$

where $C:[0, \infty) \rightarrow[0, \infty)$ is a non-decreasing function, $K \in L^{1}(\Omega)$.

And assume that

$$
F, G \geq 0 \text { and } F, G \in L^{1}(\Omega) .
$$

For the convenience of the reader we recall the definition of weak solutions we use in this paper.

Definition 2.1. We say that $(u, v)$ is a weak solution of (1) if

$$
\begin{cases}u, v \in W_{0}^{1,1}(\Omega), f(\cdot, u, v, \nabla u, \nabla v) \in L^{1}(\Omega), & \\ -\Delta u=-f(x, u, v, \nabla u, \nabla v)+F & \text { in } D^{\prime}(\Omega) \\ -\Delta v=f(x, u, v, \nabla u, \nabla v)+G & \text { in } D^{\prime}(\Omega) .\end{cases}
$$

We will be interested in proving the existence of weak positive solutions of problem (1).

Theorem 2.2. Under hypotheses (6)-(8), system (1) has a positive weak solution.

Remark 2.3. (a) It should be noted that there is no growth restriction on the "lower order nonlinearity" of $f$ as a function of $u$ and $v$ and the growth of $f$ with respect to the gradient can be quadratic. Hence the present theorem extends some results in [3] and in [16].

(b) The result of this work include the typical model (4) and the more general one

$$
\begin{cases}-\Delta u=-(u+v)\left[|\nabla u|^{2}+|\nabla v|^{2}\right]+F(x) & \text { in } \Omega \\ -\Delta v=(u+v)\left[|\nabla u|^{2}+|\nabla v|^{2}\right]+G(x) & \text { in } \Omega \\ u=v=0 & \text { on } \partial \Omega .\end{cases}
$$




\section{Proof of Theorem 2.2}

As explained before, Theorem 2.2 will be proved if we show the existence of solutions for (3). To do this, we will need the following functions. Let $H$ be a function in $C^{1}(\mathbb{R})$, such that $0 \leq H(s) \leq 1$, and

$$
H(s)= \begin{cases}0 & \text { if }|s| \geq 1 \\ 1 & \text { if }|s| \leq \frac{1}{2}\end{cases}
$$

And for a given real positive number $k$, we define the function

$$
T_{k}(s)=\max \{-k, \min (s, k)\}, \quad \text { for } s \in \mathbb{R} .
$$

\subsection{Approximating scheme.}

In this paragraph, we define an approximated equation of (3) by truncating $F$ and $G$ as follows: for $n \geq 1$, we choose $F_{n}$ and $G_{n}$ nonnegative functions such that

$$
\begin{aligned}
& F_{n}, G_{n} \in L^{\infty}(\Omega) \\
& \left\|F_{n}\right\|_{L^{1}(\Omega)} \leq\|F\|_{L^{1}(\Omega)},\left\|G_{n}\right\|_{L^{1}(\Omega)} \leq\|G\|_{L^{1}(\Omega)}, \\
& F_{n} \longrightarrow F, G_{n} \longrightarrow G, \text { strongly in } L^{1}(\Omega) \text { as } n \rightarrow \infty .
\end{aligned}
$$

We then consider the following linear problem:

$$
\left\{\begin{array}{l}
-\Delta w_{n}=F_{n}+G_{n} \quad \text { in } D^{\prime}(\Omega), \\
w_{n} \in W_{0}^{1, \infty}(\Omega) .
\end{array}\right.
$$

It is well known that (10) has a positive solution $w_{n}$ and there exists $w$, up to a subsequence still denoted by $w_{n}$, such that

$$
\begin{aligned}
& w_{n} \rightarrow w \text { strongly in } W_{0}^{1, q}(\Omega), 1 \leq q<\frac{N}{N-1} \\
& w_{n} \rightarrow w \text { a.e. in } \Omega \\
& \nabla w_{n} \rightarrow \nabla w \text { a.e. in } \Omega .
\end{aligned}
$$

We also define the nonlinear function $f_{n}$ by:

$$
f_{n}(x, u, \nabla u)=f\left(x, u, w_{n}-u, \nabla u, \nabla w_{n}-\nabla u\right) \rho_{n}(u)
$$

where $\rho_{n}(x)=x^{2} /\left(x^{2}+n\right)$, then $\rho_{n}(0)=0, \lim _{n \rightarrow \infty} \rho_{n}(x)=0$, and $0 \leq$ $\rho_{n} \leq 1$.

One can see that $f_{n}$ satisfies the same hypotheses as $f$, especially hypotheses (6)-(8). We remark here that (7) can be reformulated by

$$
\begin{aligned}
\left|f_{n}(x, u, \nabla u)\right| & \leq C\left(|u|+\left|w_{n}-u\right|\right)\left(|\nabla u|^{2}+\left|\nabla\left(w_{n}-u\right)\right|^{2}+K(x)\right) \\
& \leq C_{1}\left(u+w_{n}\right)\left(|\nabla u|^{2}+\left|\nabla w_{n}\right|^{2}+K(x)\right) .
\end{aligned}
$$


We consider now the approximating problem

$$
\left\{\begin{array}{l}
-\Delta u_{n}+f_{n}\left(x, u_{n}, \nabla u_{n}\right)=F_{n} \quad \text { in } D^{\prime}(\Omega), \\
u_{n} \in W_{0}^{1, \infty}(\Omega) .
\end{array}\right.
$$

To show that equation (12) has a solution, one can see that $u_{1}=0$ is a subsolution and $u_{2}=w_{n}$ is a supersolution. Then by virtue of the classical results in [14], [10], [5] there exists $u_{n}$ solution of (12) such that

$$
0 \leq u_{n} \leq w_{n} \text { for all } n \text {. }
$$

\subsection{Estimates.}

Lemma 3.2.1. Let $u_{n}$ and $f_{n}$ be sequences defined as above. Then

$$
\begin{aligned}
& \text { (i) } \int_{\Omega} f_{n}\left(x, u_{n}, \nabla u_{n}\right) d x \leq\|F\|_{L^{1}(\Omega)}, \\
& \text { (ii) } \int_{\Omega}\left|\nabla T_{k}\left(u_{n}\right)\right|^{2} d x \leq k\|F\|_{L^{1}(\Omega)} .
\end{aligned}
$$

Proof. (i) Integrating (12) over $\Omega$, we obtain

$$
-\int_{\Omega} \Delta u_{n}+\int_{\Omega} f_{n}=\int_{\Omega} F_{n} .
$$

On the other hand, it is well known that for every function $y$ in $W_{0}^{1.1}(\Omega)$ such that

$$
\left\{\begin{array}{l}
-\Delta y=H, \quad H \in L^{1}(\Omega) \\
y \geq 0
\end{array}\right.
$$

there exists a sequence $y_{n}$ in $C^{2}(\Omega) \cap C_{0}(\bar{\Omega})$ which satisfies

$$
\begin{aligned}
y_{n} & \longrightarrow y \text { strongly in } W_{0}^{1.1}(\Omega) \\
\Delta y_{n} & \longrightarrow \Delta y \text { strongly in } L^{1}(\Omega) .
\end{aligned}
$$

The regularity of $y_{n}$ allows us to write

$$
\int_{\Omega} \Delta y_{n}=\int_{\partial \Omega} \frac{\partial y_{n}}{\partial \nu} d \sigma .
$$

But $y_{n} \geq 0$ on $\Omega$ and $y_{n}=0$ on $\partial \Omega$, then $\frac{\partial y_{n}}{\partial \nu} \leq 0$. We deduce by passing to the limit that $\int_{\Omega} \Delta y \leq 0$. Therefore

$$
\int_{\Omega} \Delta u_{n} \leq 0
$$


Then relation (14) yields

$$
\int_{\Omega} f_{n} \leq \int_{\Omega} F_{n} \leq\|F\|_{L^{1}(\Omega)} .
$$

(ii) Multiplying the equation in (12) by $T_{k}\left(u_{n}\right)$ and integrating over $\Omega$, we obtain

$$
\int_{\Omega}\left|\nabla T_{k}\left(u_{n}\right)\right|^{2}+\int_{\Omega} f_{n} T_{k}\left(u_{n}\right)=\int_{\Omega} F_{n} T_{k}\left(u_{n}\right) .
$$

The nonnegativity of $f_{n}$ and $T_{k}\left(u_{n}\right)$ and the hypotheses on $F_{n}$ allow us to write

$$
\int_{\Omega}\left|\nabla T_{k}\left(u_{n}\right)\right|^{2} \leq k\|F\|_{L^{1}(\Omega)} .
$$

Remark 3.2.2. (a) Assertion $(i)$ and the compactness of the operator defined from $L^{1}(\Omega)$ into $W_{0}^{1, q}(\Omega), 1 \leq q<N /(N-1)$, by: $g \mapsto v$ where $v$ is a solution of the problem

$$
\left\{\begin{array}{l}
-\Delta v=g \quad \text { in } \mathfrak{D}^{\prime}(\Omega) \\
v \in W_{0}^{1, q}(\Omega)
\end{array}\right.
$$

imply the existence of $u$, up to a subsequence still denoted by $u_{n}$ for simplicity, such that $u_{n}$ converges strongly to $u$, in $W_{0}^{1, q}(\Omega), 1 \leq q<N /(N-1)$, and $\left(u_{n}, \nabla u_{n}\right)$ converges almost everywhere in $\Omega$ (see [9]).

(b) Assertion (ii) and (a) imply that $T_{k}\left(u_{n}\right)$ converges weakly to $T_{k}(u)$ in $H_{0}^{1}(\Omega)$.

Lemma 3.2.3. Let $w_{n}$ (respectively $u_{n}$ ) be solutions of (10) (respectively (12)), then

(i) $\lim _{h \rightarrow \infty} \frac{1}{h} \int_{\Omega}\left|\nabla T_{h}\left(u_{n}\right)\right|^{2}=0, \lim _{h \rightarrow \infty} \frac{1}{h} \int_{\Omega}\left|\nabla T_{h}\left(w_{n}\right)\right|^{2}=0$ uniformly on $n$.

(ii) $\lim _{n \rightarrow \infty} T_{h}\left(w_{n}\right)=T_{h}(w)$ strongly in $H_{0}^{1}(\Omega)$ for fixed positive $h$.

Proof. (i) We first remark that $u_{n}$ satisfies

$$
-\Delta u_{n} \leq F \text { in } \mathfrak{D}^{\prime}(\Omega) .
$$

If we multiply this inequality by $T_{h}\left(u_{n}\right)$ and integrate on $\Omega$, we obtain for every $0<M<h$

$$
\int_{\Omega}\left|\nabla T_{h}\left(u_{n}\right)\right|^{2} \leq \int_{\Omega \cap\left[u_{n} \leq M\right]} T_{h}\left(u_{n}\right) F+\int_{\Omega \cap\left[u_{n}>M\right]} T_{h}\left(u_{n}\right) F
$$




$$
\leq M \int_{\Omega} F+h \int_{\Omega} F \chi_{\left[u_{n}>M\right]} .
$$

Hence

$$
\frac{1}{h} \int_{\Omega}\left|\nabla T_{h}\left(u_{n}\right)\right|^{2} \leq \frac{M}{h}\|F\|_{L^{1}}+\int_{\Omega} F \chi_{\left[u_{n}>M\right]} .
$$

Fix $\varepsilon>0$. Since $u_{n}$ is bounded in $L^{1}(\Omega)$, we have

$$
\left|\left[u_{n} \geq k\right]\right|=\int_{\left[u_{n} \geq k\right]} d x \leq k^{-1}\left\|u_{n}\right\|_{L^{1}} \leq C k^{-1} .
$$

Therefore, there exists $k_{\varepsilon}$ independent of $n$ such that

$$
\int_{\Omega} F \chi_{\left[u_{n}>k_{\varepsilon}\right]} \leq \frac{\varepsilon}{2} .
$$

Taking $M=k_{\varepsilon}$ an letting $h$ tend to infinity, we obtain the desired conclusion.

For the second assertion, we remark that $w_{n}$ satisfies the same hypotheses used in the proof of the first one. Then the same arguments are still valid for $w_{n}$.

(ii) We multiply the equation in (10) by $T_{h}\left(w_{n}\right)-T_{h}(w)$ and we integrate on $\Omega$, we obtain

$$
\begin{aligned}
& \int_{\Omega}\left|\nabla T_{h}\left(w_{n}\right)-\nabla T_{h}(w)\right|^{2}+\int_{\Omega} \nabla T_{h}(w)\left(\nabla T_{h}\left(w_{n}\right)-\nabla T_{h}(w)\right) \\
& =\int_{\Omega}\left(F_{n}+G_{n}\right)\left(T_{h}\left(w_{n}\right)-T_{h}(w)\right) .
\end{aligned}
$$

We use then the fact that $\left|\left(F_{n}+G_{n}\right)\left(T_{h}\left(w_{n}\right)-T_{h}(w)\right)\right| \leq 2 h(|F|+|G|) \in$ $L^{1}(\Omega)$ and $\left(F_{n}+G_{n}\right)\left(T_{h}\left(w_{n}\right)-T_{h}(w)\right)$ converges almost everywhere to 0 in $\Omega$, to conclude by virtue of Lebesgue's theorem that

$$
\lim _{n \rightarrow \infty} \int_{\Omega}\left(F_{n}+G_{n}\right)\left(T_{h}\left(w_{n}\right)-T_{h}(w)\right)=0 .
$$

On the other hand $\nabla T_{h}\left(w_{n}\right)-\nabla T_{h}(w)$ converges weakly to 0 in $L^{2}(\Omega)$ (since $\int_{\Omega}\left|\nabla T_{h}\left(w_{n}\right)\right|^{2} \leq h\left(\|F\|_{L^{1}(\Omega)}+\|G\|_{L^{1}(\Omega)}\right)$ and $\nabla w_{n}$ converges to $\nabla w$ almost everywhere in $\Omega$ ) and $\nabla T_{h}(w) \in L^{2}(\Omega)$, consequently

$$
\lim _{n \rightarrow \infty} \int_{\Omega} \nabla T_{h}(w)\left(\nabla T_{h}\left(w_{n}\right)-\nabla T_{h}(w)\right)=0 .
$$


We conclude from (15) and (16) that

$$
\lim _{n \rightarrow \infty} \int_{\Omega}\left|\nabla T_{h}\left(w_{n}\right)-\nabla T_{h}(w)\right|^{2}=0 .
$$

\subsection{Convergence.}

The aim of this paragraph is to prove that $u$ (obtained in the previous section) is in fact a solution of problem (12). According to Definition 2.1, we have only to show that

$$
-\Delta u+f(x, u, w-u, \nabla u, \nabla w-\nabla u)=F \quad \text { in } \mathfrak{D}^{\prime}(\Omega) .
$$

We know by Lemma 3.2.1 that $f_{n}$ is uniformly bounded in $L^{1}(\Omega)$. Moreover $f_{n} \geq 0$ and for almost every $x$ in $\Omega$

$$
\lim _{n \rightarrow \infty} f_{n}\left(x, u_{n}, \nabla u_{n}\right)=f(x, u, w-u, \nabla u, \nabla w-\nabla u) .
$$

Then there exists $\mu$ a non-negative measure (see [22]) such that

$$
\begin{aligned}
\lim _{n \rightarrow \infty}\left(-\Delta u_{n}+f_{n}\left(x, u_{n}, \nabla u_{n}\right)\right)= & -\Delta u+f(x, u, w-u, \nabla u, \nabla w-\nabla u) \\
& +\mu \text { in } \mathfrak{D}^{\prime}(\Omega) .
\end{aligned}
$$

On the other hand

$$
\lim _{n \rightarrow \infty}\left(-\Delta u_{n}+f_{n}\left(x, u_{n}, \nabla u_{n}\right)\right)=\lim _{n \rightarrow \infty} F_{n}=F \quad \text { in } L^{1}(\Omega) .
$$

Consequently

$$
-\Delta u+f(x, u, w-u, \nabla u, \nabla w-\nabla u) \leq F \quad \text { in } \mathfrak{D}^{\prime}(\Omega) .
$$

Therefore to conclude the proof of Theorem 2.2, we must establish the opposite inequality. To this end we introduce the following test function

$$
\psi \exp \left(-C_{2}\left(u_{n}+w_{n}\right)\right) H\left(\frac{u_{n}}{k}\right) H\left(\frac{w_{n}}{k}\right),
$$

where $H$ denotes the function defined above, $C_{2}(s)=\int_{0}^{s} C_{1}(t) d t\left(C_{1}\right.$ is given by relation (11) ) and $\psi \leq 0, \psi \in H_{0}^{1}(\Omega) \cap L^{\infty}(\Omega)$. We multiply the equation satisfied by $u_{n}$ in (12) by this test function and we integrate on $\Omega$, we obtain

$$
\int_{\Omega} F_{n} \psi \exp \left(-C_{2}\left(u_{n}+w_{n}\right)\right) H\left(\frac{u_{n}}{k}\right) H\left(\frac{w_{n}}{k}\right)=I_{1}+I_{2}+I_{3}+I_{4}+I_{5},
$$

where

$$
I_{1}=\int_{\Omega} \nabla u_{n} \nabla \psi \exp \left(-C_{2}\left(u_{n}+w_{n}\right)\right) H\left(\frac{u_{n}}{k}\right) H\left(\frac{w_{n}}{k}\right)
$$




$$
\begin{aligned}
& I_{2}=-\int_{\Omega} \psi \nabla u_{n} \nabla w_{n} C_{1}\left(u_{n}+w_{n}\right) \exp \left(-C_{2}\left(u_{n}+w_{n}\right)\right) H\left(\frac{u_{n}}{k}\right) H\left(\frac{w_{n}}{k}\right) \\
& I_{3}=\frac{1}{k} \int_{\Omega}\left|\nabla u_{n}\right|^{2} \psi \exp \left(-C_{2}\left(u_{n}+w_{n}\right)\right) H^{\prime}\left(\frac{u_{n}}{k}\right) H\left(\frac{w_{n}}{k}\right) \\
& I_{4}=\frac{1}{k} \int_{\Omega} \nabla u_{n} \nabla w_{n} \psi \exp \left(-C_{2}\left(u_{n}+w_{n}\right)\right) H\left(\frac{u_{n}}{k}\right) H^{\prime}\left(\frac{w_{n}}{k}\right) \\
& I_{5}=\int_{\Omega}\left(f_{n}-\left|\nabla u_{n}\right|^{2} C_{1}\left(u_{n}+w_{n}\right)\right) \psi \exp \left(-C_{2}\left(u_{n}+w_{n}\right)\right) H\left(\frac{u_{n}}{k}\right) H\left(\frac{w_{n}}{k}\right) .
\end{aligned}
$$

By investigating separately each term, we get for the first one

$$
\begin{aligned}
\lim _{n \rightarrow \infty} I_{1} & =\lim _{n \rightarrow \infty} \int_{\Omega} \nabla T_{k}\left(u_{n}\right) \nabla \psi \exp \left(-C_{2}\left(u_{n}+w_{n}\right)\right) H\left(\frac{u_{n}}{k}\right) H\left(\frac{w_{n}}{k}\right) \\
& =\int_{\Omega} \nabla u \nabla \psi \exp \left(-C_{2}(u+w)\right) H\left(\frac{u}{k}\right) H\left(\frac{w}{k}\right),
\end{aligned}
$$

since

$$
\nabla \psi \exp \left(-C_{2}\left(u_{n}+w_{n}\right)\right) H\left(\frac{u_{n}}{k}\right) H\left(\frac{w_{n}}{k}\right)
$$

converges strongly to

$$
\nabla \psi \exp \left(-C_{2}(u+w)\right) H\left(\frac{u}{k}\right) H\left(\frac{w}{k}\right) \text { in } L^{2}(\Omega)
$$

and $\nabla T_{k}\left(u_{n}\right)$ converges weakly to $\nabla T_{k}(u)$ in $L^{2}(\Omega)$, see [15, Lemma 1.3 , p. $12]$. Concerning the second term, we first remark that

$$
I_{2}=-\int_{\Omega} \psi \nabla T_{k}\left(u_{n}\right) \nabla T_{k}\left(w_{n}\right) C_{1}\left(u_{n}+w_{n}\right) \exp \left(-C_{2}\left(u_{n}+w_{n}\right)\right) H\left(\frac{u_{n}}{k}\right) H\left(\frac{w_{n}}{k}\right) .
$$

Then by using the same argument, we have

$$
\lim _{n \rightarrow \infty} I_{2}=-\int_{\Omega} \psi \nabla u \nabla w C_{1}(u+w) \exp \left(-C_{2}(u+w)\right) H\left(\frac{u}{k}\right) H\left(\frac{w}{k}\right),
$$

since

$$
\psi \nabla T_{k}\left(w_{n}\right) C_{1}\left(u_{n}+w_{n}\right) \exp \left(-C_{2}\left(u_{n}+w_{n}\right)\right) H\left(\frac{u_{n}}{k}\right) H\left(\frac{w_{n}}{k}\right)
$$

converges to

$$
\psi \nabla T_{k}(w) C_{1}(u+w) \exp \left(-C_{2}(u+w)\right) H\left(\frac{u}{k}\right) H\left(\frac{w}{k}\right)
$$

strongly in $L^{2}(\Omega)$ as $n$ tends to infinity and $\nabla T_{k}\left(u_{n}\right)$ converges weakly to $\nabla T_{k}(u)$ in $L^{2}(\Omega)$, see [15, Lemma 1.3, p. 12]. 
In order to deal with $I_{3}$ and $I_{4}$ we use Lemma $3.2 .3(i)$. We have

$$
I_{3} \leq\|\psi\|_{L^{\infty}(\Omega)} \frac{1}{k} \int_{\Omega}\left|\nabla T_{k}\left(u_{n}\right)\right|^{2},
$$

since $\exp \left(-C_{2}\left(u_{n}+w_{n}\right)\right) \leq 1$. Then

$$
\lim _{k \rightarrow \infty} I_{3}=0 \text { uniformly on } n .
$$

For $I_{4}$ we first use Holder's inequality to write

$$
\begin{aligned}
I_{4} \leq & \frac{1}{k}\left(\int_{\Omega}\left|\nabla T_{k}\left(u_{n}\right)\right|^{2} \mid \psi \exp \left(-C_{2}\left(u_{n}+w_{n}\right) H\left(\frac{u_{n}}{k}\right) H^{\prime}\left(\frac{w_{n}}{k}\right) \mid\right)^{1 / 2}\right. \\
& \times \frac{1}{k}\left(\int_{\Omega}\left|\nabla T_{k}\left(w_{n}\right)\right|^{2} \mid \psi \exp \left(-C_{2}\left(u_{n}+w_{n}\right) H\left(\frac{u_{n}}{k}\right) H^{\prime}\left(\frac{w_{n}}{k}\right) \mid\right)^{1 / 2}\right. \\
\leq & \left(\|\psi\|_{L^{\infty}(\Omega)} \frac{1}{k} \int_{\Omega}\left|\nabla T_{k}\left(u_{n}\right)\right|^{2}\right)^{1 / 2}\left(\|\psi\|_{L^{\infty}(\Omega)} \frac{1}{k} \int_{\Omega}\left|\nabla T_{k}\left(w_{n}\right)\right|^{2}\right)^{1 / 2} .
\end{aligned}
$$

Thus

$$
\lim _{k \rightarrow \infty} I_{4}=0 \text { uniformly on } n \text {. }
$$

Now we investigate the remaining term $I_{5}$. Since $f_{n}$ satisfies the inequality (11) and $\psi \leq 0$, we have

$$
\begin{aligned}
& \left(f_{n}-\left|\nabla u_{n}\right|^{2} C_{1}\left(u_{n}+w_{n}\right)\right) \psi \exp \left(-C_{2}\left(u_{n}+w_{n}\right)\right) H\left(\frac{u_{n}}{k}\right) H\left(\frac{w_{n}}{k}\right) \\
& \geq \psi \exp \left(-C_{2}\left(u_{n}+w_{n}\right)\right) H\left(\frac{u_{n}}{k}\right) H\left(\frac{w_{n}}{k}\right) C_{1}\left(u_{n}+w_{n}\right)\left(\left|\nabla T_{k}\left(w_{n}\right)\right|^{2}+K(x)\right) .
\end{aligned}
$$

Therefore by applying Fatou's lemma, we obtain

$$
\lim _{n \rightarrow \infty} I_{5} \geq \int_{\Omega}\left(f-|\nabla u|^{2} C_{1}(u+w)\right) \psi \exp \left(-C_{2}(u+w)\right) H\left(\frac{u}{k}\right) H\left(\frac{w}{k}\right) .
$$

On the other hand we have by Lebesgue's theorem

$$
\begin{aligned}
& \lim _{n \rightarrow \infty} \int_{\Omega} F_{n} \psi \exp \left(-C_{2}\left(u_{n}+w_{n}\right)\right) H\left(\frac{u_{n}}{k}\right) H\left(\frac{w_{n}}{k}\right) \\
& =\int_{\Omega} F \psi \exp \left(-C_{2}(u+w)\right) H\left(\frac{u}{k}\right) H\left(\frac{w}{k}\right) .
\end{aligned}
$$


Finally we have shown

$$
\begin{aligned}
& \int_{\Omega} \nabla u \nabla \psi \exp \left(-C_{2}(u+w)\right) H\left(\frac{u}{k}\right) H\left(\frac{w}{k}\right) \\
& +\int_{\Omega} \psi\left(f-C_{1}(u+w)|\nabla u|^{2} u\right) \exp \left(-C_{2}(u+w)\right) H\left(\frac{u}{k}\right) H\left(\frac{w}{k}\right)+\omega\left(\frac{1}{k}\right) \\
& -\int_{\Omega} \psi \nabla u \nabla w C_{1}(u+w) \exp \left(-C_{2}(u+w)\right) H\left(\frac{u}{k}\right) H\left(\frac{w}{k}\right) \\
& \leq \int_{\Omega} F \psi \exp \left(-C_{2}(u+w)\right) H\left(\frac{u}{k}\right) H\left(\frac{w}{k}\right),
\end{aligned}
$$

where $\omega(\varepsilon)$ denotes a quantity that tends to 0 when $\varepsilon$ tends to 0 . Now we choose

$$
\psi=-\varphi \exp \left(C_{2}(u+w)\right) H\left(\frac{u}{k}\right) H\left(\frac{w}{k}\right),
$$

where $\varphi \geq 0, \varphi \in \mathfrak{D}(\Omega)$ and we replace $\psi$ by this value in the previous inequality to get

$$
\begin{aligned}
& -\int_{\Omega} \nabla u \nabla \varphi H^{2}\left(\frac{u}{k}\right) H^{2}\left(\frac{w}{k}\right)-\int_{\Omega} \nabla u \nabla(u+w) C_{1}(u+w) \varphi H^{2}\left(\frac{u}{k}\right) H^{2}\left(\frac{w}{k}\right) \\
& -\frac{1}{k} \int_{\Omega}|\nabla u|^{2} \varphi H\left(\frac{u}{k}\right) H^{\prime}\left(\frac{u}{k}\right) H^{2}\left(\frac{w}{k}\right) \\
& -\frac{1}{k} \int_{\Omega} \nabla u \nabla w \varphi H^{2}\left(\frac{u}{k}\right) H^{\prime}\left(\frac{w}{k}\right) H\left(\frac{w}{k}\right)+\omega\left(\frac{1}{k}\right) \\
& -\int_{\Omega} \varphi\left(f-C_{1}(u+w)|\nabla u|^{2}\right) H\left(\frac{u}{k}\right)^{2} H^{2}\left(\frac{w}{k}\right) \\
& +\int_{\Omega} \varphi \nabla u \nabla w C_{1}(u+w) H^{2}\left(\frac{u}{k}\right) H^{2}\left(\frac{w}{k}\right) \\
& \leq-\int_{\Omega} F \varphi H^{2}\left(\frac{u}{k}\right) H^{2}\left(\frac{w}{k}\right) .
\end{aligned}
$$

By developing calculations and remarking that the second and the third terms are equivalent to $\omega\left(\frac{1}{k}\right)$, we can write

$$
-\int_{\Omega} \nabla u \nabla \varphi H^{2}\left(\frac{u}{k}\right) H^{2}\left(\frac{w}{k}\right)-\int_{\Omega} \varphi f H\left(\frac{u}{k}\right)^{2} H^{2}\left(\frac{w}{k}\right)+\omega\left(\frac{1}{k}\right)
$$




$$
\leq-\int_{\Omega} F \varphi H^{2}\left(\frac{u}{k}\right) H^{2}\left(\frac{w}{k}\right) .
$$

We finally pass to the limit as $k$ tends to infinity and we use the fact that

$$
\lim _{k \rightarrow \infty} H\left(\frac{u}{k}\right)=1 \text { and } \lim _{k \rightarrow \infty} H\left(\frac{w}{k}\right)=1,
$$

to conclude that for every $\varphi \geq 0, \varphi \in \mathfrak{D}(\Omega)$ that

$$
\int_{\Omega} \nabla u \nabla \varphi+\int_{\Omega} \varphi f \geq \int_{\Omega} F \varphi .
$$

This finishes the proof of Theorem 2.2.

Acknowledgments. The authors would like to thank Professor Michel Pierre for his valuable discussions during his visits to Marrakech.

\section{References}

[1] Alaa, N., Solutions faibles d'équations paraboliques quasi-linéaires avec données initiales mesures, Ann. Math. Blaise Pascal 3(2) (1996), 1-15.

[2] Alaa, N., Mounir, I., Global existence for reaction-diffusion systems with mass control and critical growth with respect to the gradient, J. Math. Anal. Appl. 253 (2001), $532-557$.

[3] Alaa, N., Mounir, I., Weak solutions for some reaction-diffusion systems with balance law and critical growth with respect to the gradient, Ann. Math. Blaise Pascal 8(2) (2001), 1-19.

[4] Alaa, N., Pierre, M., Weak solution of some quasilinear elliptic equations with measures, SIAM J. Math. Anal. 24(1) (1993), 23-35.

[5] Amann, H., Crandall, M. G., On some existence theorems for semi linear equations, Indiana Univ. Math. J. 27 (1978), 779-790.

[6] Bensoussan, A., Boccardo, L., Murat, F., On a non linear partial differential equation having natural growth terms and unbounded solution, Ann. Inst. H. Poincaré Anal. Non Linéaire 5(4) (1988), 347-364.

[7] Boccardo, L., Murat, F., Puel, J. P., Existence de solutions non bornées pour certaines équations quasi-linéaires, Portugal. Math. 41 (1982), 507-534.

[8] Boudiba, N., Existence globale pour des systèmes de réaction-diffusion avec controle de masse, Ph.D. thesis, Université de Rennes I, France, 1999.

[9] Brezis, H., Strauss, W., Semilinear elliptic equation in $L^{1}$, J. Math. Soc. Japan 25 (1973), 565-590.

[10] Choquet-Bruhat, Y., Leray, J., Sur le problème de Dirichlet quasilinéaire d'ordre deux, C. R. Acad. Sci. Paris Ser. I Math. 274 (1972), 81-85.

[11] Fitzgibbon, W. E., Morgan, J., Existence of solutions for a class of weakly coupled semilinear elliptic systems, J. Differential Equations 77 (1989), 351-368.

[12] Fitzgibbon, W. E., Morgan, J., Sanders, R., Global existence and boundedness for class of inhomogeneous semilinear parabolic systems, Nonlinear Anal. 19(9) (1992), 885-899. 
[13] Hollis, S. L., Martin, R. H., Pierre, M., Global existence and boundeness in reactiondiffusion systems, SIAM. J. Math. Anal. 18 (1987), 744-761.

[14] Lions, P. L., Résolution de problèmes elliptiques quasilinéaires, Arch. Rational Mech. Anal. 74 (1980), 335-353.

[15] Lions, J. L., Quelques méthodes de résolutions des problèmes aux limites non linéaires, Dunod; Gauthier Villars, Paris, 1969.

[16] Maach, F., Existence pour des systèmes de réaction-diffusion quasi-linéaires avec loi de balance, Ph.D. thesis, Université Henri Poincaré, Nancy I, France, 1994.

[17] Pierre, M., An $L^{1}$-method to prove global existence in some reaction-diffusion systems, in "Contribution to Nonlinear Partial Differential Equations", Vol. II (Paris, 1985), Pitman Res. Notes Math. Ser. 155 (1987), 220-231.

[18] Pierre, M., Schmitt, D., Blow up in reaction-diffusion systems with dissipation of mass, SIAM J. Math. Anal. 28(2) (1987), 259-269.

[19] Pierre, M., Schmitt, D., Blow up in reaction-diffusion systems with dissipation of mass, SIAM Rev. 42(1) (2000), 93-106.

[20] Porretta, A., Existence for elliptic equations in $L^{1}$ having lower order terms with natural growth, Portugal. Math. 57(2) (2000), 179-190.

[21] Rothe, F., Global Solutions of Reaction-Diffusion Systems, Lectures Notes in Math. 1072 (1984), Springer, New York.

[22] Schwartz, J. T., Nonlinear Functional Analysis, Gordon and Breach Science Publishers, New York-London, Paris, 1969.

Nour Eddine AlaA

FACUlTÉ DES SCIENCES

et Techniques Gueliz

DÉPARTEMENT DE MATHÉMATIQUES

ET INFORMATIQUE

B. P. 618 Marrakech, Maroc

E-MAIL: ALAA@FSTG-MARRAKECH.AC.MA

ILHAM MOUNIR

Université Cadi Ayyad

Faculté des Sciences Semlalia

DÉPARTEMENT De MAThÉmatiques

Prince My Abdellah B. P. 511

MARRAKECH, MAROC

E-MAIL: ILHAMOUNIR@UCAM.AC.MA
F. MAACH

FACulté Des ScIEnCES et Techniques Gueliz DÉPARTEMENT DE MATHÉMATIQUES

ET INFORMATIQUE

B. P. 618 Marrakech, Maroc 